\title{
Geometric Power Lindley Poisson Distribution: Properties and Applications
}

\author{
Mahmoud M. Mansour \\ Department of Statistics, Mathematics and Insurance, \\ Benha University, Egypt \\ Mahmoud.mansour@fcom.bu.edu.eg \\ Mohammad Ahsanullah \\ Department of Management Sciences, Rider University \\ Lawrenceville, NJ 08648-3009 \\ ahsan@rider.edu \\ Zohdy M. Nofal \\ Department of Statistics, Mathematics and Insurance, \\ Benha University, Egypt \\ dr_znofal@hotmail.com \\ Omar H. Khalaf \\ Department of Statistics, Mathematics and Insurance, \\ Benha University, Egypt
}

Received 10 January 2016

Accepted 12 October 2016

\begin{abstract}
In this Paper, a new four-parameter distribution motivated mainly by dealing with series-parallel system is introduced. Moments, conditional moments and moment generating function of the new distribution including are presented. Estimation of its parameters is studied. characterization of the new model is introduced.

Keywords: Lindley distribution; Geometric distribution; Maximum likelihood estimation; truncated Poisson distribution.

2000 Mathematics Subject Classification: 60-XX, 60EXX.
\end{abstract}

\section{Introduction}

The quality of the procedures used in a statistical analysis depends heavily on the assumed probability model or distributions. In view of it, extensive exertion has been used in the improvement of huge classes of standard likelihood circulations alongside applicable measurable procedures. Sankaran (1970) introduced the discrete Poisson Lindley distribution by compounding Poission and Lindley distributions. Ghitany et al. (2008) investigated the properties of the zero-truncated Poisson-Lindley distribution. Bakouch et al. (2012) extended. Lindley distribution by exponentiation. Zakerzadeh and Dolati (2010) introduced and analyzed a 
three-parameter generalization of the Lindley distribution, which was used by Mahmoudi and Zakerzadeh (2010) to derive an extended version of the compound Poisson distribution. Shanker et al. (2013) introduced a two-parameter Lindley distribution in which the one-parameter is a particular case, for modeling waiting and survival time data. Ghitany et al. (2013) introduced a two-parameter power Lindley distribution (PL) and discussed its properties. Nadarajah et al. (2011) proposed a generalized Lindley distribution (GL) and provided a comprehensive account of its mathematical properties.

Nadarajah et al. (2013) introduced a two-parameter distribution which represent a general model by taking the probability density function and the cumulative distribution function of failure times to be given by $f(x)$ and $F(x)$, respectively. Its cdf is given by

$$
G(x)=\frac{\exp (-\lambda+\lambda F(x))-e^{-\lambda}}{1-e^{-\lambda}-\delta+\delta \exp (-\lambda+\lambda F(x))}
$$

for $x>0, \lambda>0$ and $0<\delta<1$. The corresponding probability density function is,

$$
g(x)=\frac{\lambda(1-\delta)\left(1-e^{-\lambda}\right) f(x) \exp (-\lambda+\lambda F(x))}{\left\{1-e^{-\lambda}-\delta+\pi \exp (-\lambda+\lambda F(x))\right\}^{2}}
$$

Ghitany et al. (2013) introduced a new extension of the Lindley distribution called power Lindley distribution by its probability density function pdf

$$
f(x)=\frac{\theta^{2} \beta x^{\beta-1}}{\theta+1}\left(1+x^{\beta}\right) e^{-\theta x^{\beta}}, x>0, \theta, \beta>0 .
$$

The corresponding cumulative distribution function (cdf) is:

$$
F(x)=1-\frac{\theta+1+\theta x^{\beta}}{\theta+1} e^{-\theta x^{\beta}}, x>0, \theta, \beta>0 .
$$

The rest of the article is organized as follows. In Section 2, introduces the proposed new model according to geometric Poisson G-family. In Section 3, The Expansion for the pdf and the cdf Functions is derived. Moments, conditional moments and moment generating function of the new distribution including are presented in Section 4. In Section 5, we introduce Characterizations of the new model. In Section 6, we introduce the method of likelihood estimation as point estimation Finally, we fit the distribution to real data set to examine it.

\section{Geometric Power Lindley Poisson Distribution}

Using $f(x)$ and $F(x)$ as given in (3) and (4) in (1), we obtain

$$
G(x)=\frac{\exp \left(-\lambda\left(\frac{\theta+1+\theta x^{\beta}}{1+\theta}\right) e^{-\theta x^{\beta}}\right)-e^{-\lambda}}{1-e^{-\lambda}-\delta\left[1-\exp \left(-\lambda\left(\frac{\theta+1+\theta x^{\beta}}{1+\theta}\right) e^{-\theta x^{\beta}}\right)\right]^{\prime}}
$$

for $x>0, \theta>0, \lambda, \beta>0$ and $0<\delta<1$. The corresponding probability density function is, 


$$
\begin{gathered}
g(x)=\frac{\lambda \theta^{2}(1-\delta) \beta x^{\beta-1}\left(1-e^{-\lambda}\right)\left(1+x^{\beta}\right)}{(1+\theta)\left\{1-e^{-\lambda}-\delta\left[1-\exp \left(-\lambda\left(\frac{\theta+1+\theta x^{\beta}}{1+\theta}\right) e^{-\theta x^{\beta}}\right)\right]\right\}^{2}}, \\
\times \exp \left(-\theta x^{\beta}-\lambda\left(\frac{\theta+1+\theta x^{\beta}}{1+\theta}\right) e^{-\theta x^{\beta}}\right)
\end{gathered}
$$

for $x>0, \theta>0, \lambda, \beta>0$ and $0<\delta<1$. We shall refer to the distribution given by (5) and (6) as the geometric power Lindley Poisson $(G P L P)$. The parameters, $\lambda, \beta$ and $\delta$, control the shape. The parameter, $\theta$, controls the scale. The failure rate function associated with (6) is given by

$$
\begin{gathered}
h(x)=\frac{\lambda \theta^{2}(1-\delta) \beta x^{\beta-1}\left(1-e^{-\lambda}\right)\left(1+x^{\beta}\right)}{(1+\theta)\left\{1-e^{-\lambda}-\delta\left[1-\exp \left(-\lambda+\lambda\left(\frac{\theta+1+\theta x^{\beta}}{1+\theta}\right) e^{-\theta x^{\beta}}\right)\right]\right\}} \\
\times \frac{\exp \left(-\theta x^{\beta}-\lambda+\lambda\left(\frac{\theta+1+\theta x^{\beta}}{1+\theta}\right) e^{-\theta x^{\beta}}\right)}{\left(1-\exp \left(-\lambda\left(\frac{\theta+1+\theta x^{\beta}}{1+\theta}\right) e^{-\theta x^{\beta}}\right)\right)} .
\end{gathered}
$$

Also, the reversed failure rate function

$$
h_{r}(x)=\frac{\lambda \theta^{2}(1-\delta)\left(1-e^{-\lambda}\right)(1+x) \exp \left(-\theta x-\lambda\left(\frac{\theta+1+\theta x}{1+\theta}\right) e^{-\theta x}\right)}{(1+\theta)\left[\exp \left(-\lambda\left(\frac{\theta+1+\theta x}{1+\theta}\right) e^{-\theta x}\right)-e^{-\lambda}\right]\left\{1-e^{-\lambda}-\delta\left[1-\exp \left(-\lambda e^{-(\beta x)^{\alpha}}\right)\right]\right\}} .
$$

Figure 1 (a) and (b) provide some plots of the GPLP density curves for different values of the parameters $\lambda, \theta, \beta$ and $\delta$.

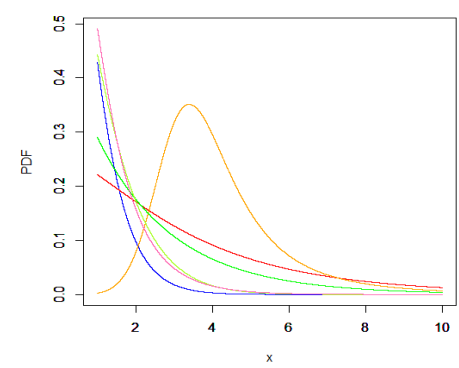

(a)

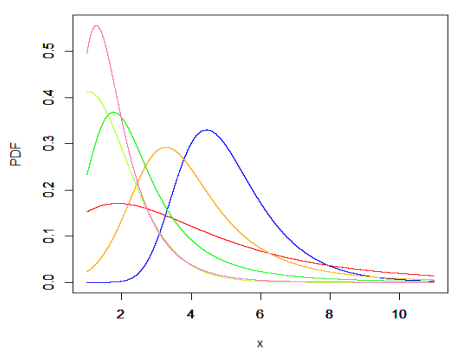

(b)

Fig. 1. Plots of the GPLP density function for some parameter values.(a) For $\lambda=0.9, \theta=2, \beta=1$ and $\delta=0.1$ (blue line), $\lambda=2, \theta=1$, $\beta=0.7$ and $\delta=0.2$ (red line), $\lambda=18, \theta=1, \beta=0.8$ and $\delta=0.9$ (orange line) $\lambda=0.5, \theta=1, \beta=0.8$ and $\delta=0.1$ (green line), $\lambda=0.5, \theta=1$, $\beta=1.2$ and $\delta=0.5$ (green yellow line), $\lambda=4, \theta=2, \beta=0.8$ and $\delta=0.5$ (hot pink line) (b) For $\lambda=30, \theta=1, \beta=1$ and $\delta=0.2$ (blue line), $\lambda=4, \theta=1, \beta=0.7$ and $\delta=0.3$ (red line), $\lambda=10, \theta=1, \beta=0.7$ and $\delta=0.3$ (orange line) $\lambda=8, \theta=1, \beta=0.8$ and $\delta=0.9$ (green line), $\lambda=1.4, \theta=1, \beta=1.2$ and $\delta=0.2$ (green yellow line), $\lambda=10, \theta=2, \beta=0.8$ and $\delta=0.5$ (hot pink line). 


\section{Expansion for the pdf and the cdf functions}

In this section we give another expression for the pdf and the cdf functions using the Maclaurin and Binomial expansions for simplifying the pdf and the cdf forms.

\subsection{Expansion for the pdf function}

We can rewrite (6) as,

$$
g(x)=\frac{\lambda \theta^{2}(1-\delta) \beta x^{\beta-1}\left(1-e^{-\lambda}\right)\left(1+x^{\beta}\right) \exp \left(-\theta x^{\beta}-\lambda\left(\frac{\theta+1+\theta x^{\beta}}{1+\theta}\right) e^{-\theta x^{\beta}}\right)}{(1+\theta)\left(1-e^{-\lambda}-\delta\right)^{2}\left[1+\frac{\pi \exp \left(-\lambda\left(\frac{\theta+1+\theta x^{\beta}}{1+\theta}\right) e^{-\theta x^{\beta}}\right)}{1-e^{-\lambda}-\delta}\right]^{2}} .
$$

We have

$$
(1-z)^{-b}=\sum_{i=0}^{\infty}\left(\begin{array}{c}
-b \\
i
\end{array}\right)(-z)^{i}, \quad|z|<1
$$

and

$$
e^{-x}=\sum_{i=0}^{\infty} \frac{(-x)^{i}}{i !}
$$

Using (9), we can write (8) as

$$
\begin{aligned}
& g(x)=\frac{\lambda \theta^{2}(1-\delta) \beta x^{\beta-1}\left(1-e^{-\lambda}\right)\left(1+x^{\beta}\right) \exp \left(-\theta x^{\beta}\right)}{(1+\theta)\left(1-e^{-\lambda}-\delta\right)^{2}} \times \\
& \sum_{k=0}^{\infty}\left(\begin{array}{c}
-2 \\
k
\end{array}\right)\left[\frac{\delta}{1-e^{-\lambda}-\delta}\right]^{k} \exp \left(-\lambda(k+1)\left(\frac{\theta+1+\theta x^{\beta}}{1+\theta}\right) e^{-\theta x^{\beta}}\right)
\end{aligned}
$$

Applying (10) to (11) for the term $\exp \left(-\lambda(k+1)\left(\frac{\theta+1+\theta x^{\beta}}{1+\theta}\right) e^{-\theta x^{\beta}}\right),(11)$ can be written as:

$$
\begin{gathered}
g(x)=\frac{\lambda \theta^{2}(1-\delta) \beta x^{\beta-1}\left(1-e^{-\lambda}\right)\left(1+x^{\beta}\right) \exp \left(-\theta x^{\beta}\right)}{(1+\theta)\left(1-e^{-\lambda}-\delta\right)^{2}} \times \\
\sum_{k, j=0}^{\infty}\left(\begin{array}{c}
-2 \\
k
\end{array}\right) \frac{(-1)^{j}}{j !}\left[\frac{\delta}{1-e^{-\lambda}-\delta}\right]^{k} \lambda^{j}(k+1)^{j}\left(1+\frac{\theta x^{\beta}}{1+\theta}\right)^{j} e^{-\theta j x^{\beta}}
\end{gathered}
$$

Applying (9) to 12) for the term $\left(1+\frac{\theta x^{\beta}}{1+\theta}\right)^{j},(12)$ can be written as: 


$$
\begin{gathered}
g(x)=\frac{\lambda \theta^{2}(1-\delta)\left(1-e^{-\lambda}\right) \beta}{(1+\theta)\left(1-e^{-\lambda}-\delta\right)^{2}} \sum_{k, j=0}^{\infty} \sum_{i=0}^{j}\left(\begin{array}{c}
j \\
i
\end{array}\right)\left(\begin{array}{c}
-2 \\
k
\end{array}\right) \frac{(-1)^{j} \theta^{i}}{j !(1+\theta)^{i}} \times \\
{\left[\frac{\pi}{1-e^{-\lambda}-\pi}\right]^{k} \lambda^{j}(k+1)^{j}\left(1+x^{\beta}\right) x^{i \beta} x^{\beta-1} e^{-(j+1) \theta x^{\beta}} .}
\end{gathered}
$$

The pdf of GPLP distribution can then be represented as:

$$
g(x)=\sum_{k, j=0}^{\infty} \sum_{i=0}^{j} A_{k: i}\left(1+x^{\beta}\right) x^{\beta(i+1)-1} e^{-(j+1) \theta x^{\beta}}
$$

Where $A_{k: i}$ is a constant term given by:

$$
A_{k: i}=\left(\begin{array}{c}
-2 \\
k
\end{array}\right)\left(\begin{array}{c}
j \\
i
\end{array}\right) \frac{\theta^{2+i}(1-\delta)\left(1-e^{-\lambda}\right)(-1)^{j} \delta^{k} \lambda^{j+1}(k+1)^{j} \beta}{(1+\theta)^{i+1}\left(1-e^{-\lambda}-\delta\right)^{k+2} j !}
$$

\subsection{Expansion for the cdf function}

We can rewrite (5) as,

$$
G(x)=\frac{\exp \left(-\lambda\left(\frac{\theta+1+\theta x^{\beta}}{1+\theta}\right) e^{-\theta x^{\beta}}\right)-e^{-\lambda}}{\left(1-e^{-\lambda}-\delta\right)\left[1+\frac{\pi \exp \left(-\lambda\left(\frac{\theta+1+\theta x^{\beta}}{1+\theta}\right) e^{-\theta x^{\beta}}\right)}{1-e^{-\lambda}-\delta}\right]}
$$

And applying the expansion in (9), the cdf function of the GPLP distribution can be written as:

$$
G(x)=\sum_{k=0}^{\infty}\left(\begin{array}{c}
-1 \\
k
\end{array}\right) \frac{\pi^{k}\left[\exp \left(-(k+1) \lambda\left(\frac{\theta+1+\theta x^{\beta}}{1+\theta}\right) e^{-\theta x^{\beta}}\right)-\exp \left(-\lambda-k \lambda\left(\frac{\theta+1+\theta x^{\beta}}{1+\theta}\right) e^{-\theta x^{\beta}}\right)\right]}{\left(1-e^{-\lambda}-\delta\right)^{k+1}} .
$$

\section{Statistical Properties}

In this section, moments, conditional moments, Moment Generating Function of the GPLP distribution.

\subsection{Moments}

The $\mathrm{r}^{\text {th }}$ non-central moments or (moments about the origin) of the GPLP under using equation (6) is given by: 


$$
\begin{gathered}
\mu_{r}=E\left(X^{r}\right)=\int_{\mathbf{0}}^{\infty} X^{r} g(x) \mathrm{d} x, \\
\mu_{r}=\int_{\mathbf{0}}^{\infty} x^{r}\left[\sum_{k, j=0}^{\infty} \sum_{i=0}^{j} A_{k: i}\left(1+x^{\beta}\right) x^{\beta(i+1)-1} e^{-(j+1) \theta x^{\beta}}\right] \mathrm{d} x,
\end{gathered}
$$

Then

$$
\begin{aligned}
& \mu_{r}=\sum_{k, j=0}^{\infty} \sum_{i=0}^{j} A_{k: i}\left[\frac{\Gamma\left(i+\frac{r}{\beta}+1\right)}{\beta[(j+1) \theta]^{\left(i+\frac{r}{\beta}+1\right)}}\right]\left[1+\frac{i+\frac{r}{\beta}+1}{(j+1) \theta}\right] \\
& E(X \mid X \leq x)=\frac{1}{\beta G(x)} \sum_{k, j=0}^{\infty} \sum_{i=0}^{j} A_{k: i}\left[\frac{\Gamma_{(j+1) \theta x^{\beta}}^{c}\left(i+\frac{r}{\beta}+1\right)}{[(j+1) \theta]^{\left(i+\frac{r}{\beta}+1\right)}}+\frac{\Gamma_{(j+1) \theta x^{\beta}}^{c}\left(i+\frac{r}{\beta}+2\right)}{[(j+1) \theta]^{\left(i+\frac{r}{\beta}+2\right)}}\right] \text {, } \\
& E(X \mid X \geq x)=\frac{1}{\beta[1-G(x)]} \sum_{k, j=0}^{\infty} \sum_{i=0}^{j} A_{k: i}\left[\frac{\Gamma_{(j+1) \theta x^{\beta}}^{c}\left(i+\frac{r}{\beta}+1\right)}{[(j+1) \theta]^{\left(i+\frac{r}{\beta}+1\right)}}+\frac{\Gamma_{(j+1) \theta x^{\beta}}^{c}\left(i+\frac{r}{\beta}+2\right)}{[(j+1) \theta]^{\left(i+\frac{r}{\beta}+2\right)}}\right],
\end{aligned}
$$

where $\Gamma_{\alpha}(n)=\int_{0}^{\alpha} x^{n-1} e^{-x} d x$ and $\Gamma_{\alpha}^{c}(n)=\int_{\alpha}^{\infty} x^{n-1} e^{-x} d x$.

\subsection{The Moment Generating Function}

The moment generating function, $M_{x}(t)$, can be easily obtained as:

$$
\begin{gathered}
M_{x}(t)=\int_{0}^{\infty} \mathrm{e}^{t x} g(x) \mathrm{d} x, \\
M_{x}(t)=\int_{0}^{\infty} \mathrm{e}^{t x} \sum_{k, j=0}^{\infty} \sum_{i=0}^{j} A_{k: i}\left(1+x^{\beta}\right) x^{\beta(i+1)+r-1} e^{-(j+1) \theta x^{\beta}} \mathrm{d} x,
\end{gathered}
$$

then, the moment generating function of the GPLP distribution is given by,

$$
M_{x}(t)=\sum_{k, j=0}^{\infty} \sum_{i=0}^{j} \frac{A_{k: i}}{[\theta(j+1)-t]^{i+2}}\{(\theta(j+1)-t) \Gamma(i+1)+\Gamma(i+2)\},
$$

then, the moment generating function of the GPLP distribution is given by, 


$$
M_{x}(t)=\sum_{k, j=0}^{\infty} \sum_{i=0}^{j} \frac{A_{k: i}}{[(j+1) \theta]^{\left(i+\frac{r}{\beta}+2\right)}}\left\{((j+1) \theta-t) \Gamma\left(i+\frac{1}{\beta}+1\right)+\Gamma\left(i+\frac{1}{\beta}+2\right)\right\} .
$$

\section{Characterization}

To prove the main theorem, we need the following two Lemmas

\section{Lemma 1.}

Suppose that $X$ is an absolutely continuous random variable with cdf $F(x)$ with $F(0)=0$ and $F(x)>0$ for all $x>0$. We assume that the pdf of $X$ as $f(x)$ and $f^{\prime}(x)$ exists for all $x>0$., For a continuous function $g(x)$ on $0<x<\infty$ with finite $E(g(x))$.if $E(g(x) \mid x \leq x)=h_{1}(x) r(x)$, where $h_{1}(x)$ is a differential function in $x>0$ and $r(x)=\frac{f(x)}{F(x)}$, then $f(x)=c \exp \left(-\int_{0}^{x} \frac{g(u)-\hat{h}(u)}{h(u)} d u\right)$ and $c$ is determned by the condition $\int_{0}^{\infty} f(x) d x=1$.

\section{Proof.}

$$
h_{1}(x)=\frac{\int_{0}^{x} g(u) f(u) d u}{f(x)}
$$

and

$$
\int_{0}^{x} g(u) f(u) d u=f(x) h_{1}(x)
$$

Differentiating the above expression, we obtain

$$
-g(x) f(x)=f(x) h_{1}{ }^{\prime}(x)+f(x) h_{1}(x)
$$

On simplification, we have

$$
\frac{f(x)}{f(x)}=\frac{g(x)+h_{1}^{\prime}(x)}{h_{1}(x)}
$$

Integrating both sides of the above equation, we obtain

$$
f(x)=c \exp \left(-\int_{0}^{x} \frac{g(x)+h_{1}^{\prime}(x)}{h_{1}(x)} d x\right)
$$

and $c$ is determned by the condition $\int_{0}^{\infty} f(x) d x=1$. 


\section{Lemma 2.}

Suppose that $X$ is an absolutely continuous random variable with $\operatorname{cdf} F(x)$ with $F(0)=0$ and $F(x)>0$ for all $x>0$. We assume that the pdf of $X$ as $f(x)$ and $f^{\prime}(x)$ exists for all $x>0$., For a continuous function $g(x)$ on $0<x<\infty$ with finite $E(g(x))$.if $E(g(x) \mid x \geq x)=h(x) r(x)$, where $h(x)$ is a differential function in $x>0$ and,$r(x)=\frac{f(x)}{1-F(x)}$, then $f(x)=c \exp \left(-\int \frac{g(x)-\hat{h}(x)}{h(x)} d x\right)$ and $c$ is determned by the condition $\int_{0}^{x} f(x) d x=1$.

\section{Proof.}

$$
h(x)=\frac{\int_{x}^{\infty} g(u) f(u) d u}{f(x)}
$$

and

$$
\int_{x}^{\infty} g(u) f(u) d u=f(x) h(x)
$$

Differentiating the above expression, we obtain

$$
g(x) f(x)=f(x) h^{\prime}(x)+f^{\prime}(x) h(x)
$$

On simplification, we have

$$
\frac{f(x)}{f(x)}=-\frac{g(x)-h^{\prime}(x)}{h(x)}
$$

Integrating both sides of the above equation, we obtain

$$
f(x)=c \exp \left(-\int_{0}^{x} \frac{g(u)-h^{\prime}(u)}{h(u)} d u\right)
$$

and $c$ is determned by the condition $\int_{0}^{\infty} f(x) d x=1$.

\section{Theorem 1.}

Suppose that $\mathrm{X}$ is an absolutely continuous random variable with $\operatorname{cdf} G(x)$ with $G(0)=0$ and $G(x)>0$ for all $x>0$. We assume that the pdf of $X$ as $g(x)$ and $g^{\prime}(x)$ exists for all $x>0$.

We assume $E\left(X^{r}\right)$ exists for $r \geq 1$. then $E\left(X^{r} \mid X \leq x\right)=h_{0}(x) \tau(x)$, where $\tau(x)=\frac{g(x)}{G(x)}$ and $h_{0}(x)=\frac{A(x)}{B(x)}$ 
where $A(x)=\sum_{k, j=0}^{\infty} \sum_{i=0}^{j} A_{k: i}\left[\frac{\Gamma_{(j+1) \theta x^{\beta}}\left(i+\frac{r}{\beta}+1\right)}{\beta[(j+1) \theta]^{\left(i+\frac{r}{\beta}+1\right)}}+\frac{\Gamma_{(j+1) \theta x^{\beta}}\left(i+\frac{r}{\beta}+2\right)}{\beta[(j+1) \theta]^{\left(i+\frac{r}{\beta}+2\right)}}\right]$

$B(x)=g(x)$ and $A_{k: i}$ is given before

if and only if $g(x)=\sum_{k, j=0}^{\infty} \sum_{i=0}^{j} A_{k: i}\left(1+x^{\beta}\right) x^{\beta(i+1)-1} e^{-(j+1) \theta x^{\beta}}$

\section{Proof.}

We have

$$
\begin{aligned}
g(x) h_{0}(x) & =\int_{0}^{x} u^{r} g(u) d u \\
& =\sum_{k, j=0}^{\infty} \sum_{i=0}^{j} A_{k: i}\left[\frac{\Gamma_{(j+1) \theta x^{\beta}}\left(i+\frac{r}{\beta}+1\right)}{\beta[(j+1) \theta]^{\left(i+\frac{r}{\beta}+1\right)}}+\frac{\Gamma_{(j+1) \theta x^{\beta}}\left(i+\frac{r}{\beta}+2\right)}{\beta[(j+1) \theta]^{\left(i+\frac{r}{\beta}+2\right)}}\right] \\
& =A(x)
\end{aligned}
$$

where $\Gamma_{\alpha}(\beta)=\int_{\alpha}^{\infty} x^{\beta-1} e^{-x} d x$

Suppose that $h_{0}(x)=\frac{A(x)}{g(x)}$, then

$$
\begin{gathered}
h_{0}^{\prime}(x)=x^{r}-\frac{A(x)}{(g(x))^{2}} g^{\prime}(x) \\
h_{0}^{\prime}(x)=x^{r}-h_{0}(x) \frac{\sum_{k, j=0}^{\infty} \sum_{i=0}^{j} A_{k: i}\left(x^{\beta(i+1)-1} x^{\beta(i+1)-2}+\left(x^{\beta(i+2)-1}\right) x^{\beta(i+2)-2}-(j+1) \theta \beta x^{\beta-1}\right) e^{-(j+1) \theta x^{\beta}}}{\sum_{k, j=0}^{\infty} \sum_{i=0}^{j} A_{k: i}\left(x^{\beta(i+1)-1}+x^{\beta(i+2)-1}\right) e^{-(j+1) \theta x^{\beta}}}
\end{gathered}
$$

Thus

$$
\frac{x^{r}-h_{0}^{\prime}(x)}{h_{0}(x)}=\frac{\sum_{k, j=0}^{\infty} \sum_{i=0}^{j} A_{k: i}\left(x^{\beta(i+1)-1} x^{\beta(i+1)-2}+\left(x^{\beta(i+2)-1}\right) x^{\beta(i+2)-2}-(j+1) \theta \beta x^{\beta-1}\right) e^{-(j+1) \theta x^{\beta}}}{\sum_{k, j=0}^{\infty} \sum_{i=0}^{j} A_{k: i}\left(x^{\beta(i+1)-1}+x^{\beta(i+2)-1}\right) e^{-(j+1) \theta x^{\beta}}}
$$

Integrating both sides of the above equation we obtain

$$
\begin{aligned}
g(x) & =c \exp \int\left(\frac{\sum_{k, j=0}^{\infty} \sum_{i=0}^{j} A_{k: i}\left(x^{\beta(i+1)-1} x^{\beta(i+1)-2}+\left(x^{\beta(i+2)-1}\right) x^{\beta(i+2)-2}-(j+1) \theta \beta x^{\beta-1}\right) e^{-(j+1) \theta x^{\beta}}}{\sum_{k, j=0}^{\infty} \sum_{i=0}^{j} A_{k: i}\left(x^{\beta(i+1)-1}+x^{\beta(i+2)-1}\right) e^{-(j+1) \theta x^{\beta}}}\right) d x \\
& =c \sum_{k, j=0}^{\infty} \sum_{i=0}^{j} A_{k: i}\left(x^{\beta(i+1)-1}+x^{\beta(i+2)-1}\right) e^{-(j+1) \theta x^{\beta}}, \text { where } \mathrm{c} \text { is a constant }
\end{aligned}
$$


and

$$
\frac{1}{c}=\int_{0}^{\infty} \sum_{k, j=0}^{\infty} \sum_{i=0}^{j} A_{k: i}\left(x^{\beta(i+1)-1}+x^{\beta(i+2)-1}\right) e^{-(j+1) \theta x^{\beta}}
$$

\section{Theorem 2.}

Suppose that $\mathrm{X}$ is an absolutely continuous random variable with $\operatorname{cdf} G(x)$ with $G(0)=0$ and $G(x)>0$ for all $x>0$. We assume that the pdf of $X$ as $g(x)$ and $g^{\prime}(x)$ exists for all $x>0$.

We assume $E\left(X^{r m}\right)$ exists for $r \geq 1$.then $E\left(X^{r} \mid X \geq x\right)=h_{1}(x) \tau(x)$, where $\tau(x)=\frac{g(x)}{1-G(x)}$ and $h_{1}(x)=$ $\frac{A_{1}(x)}{B(x)}$

where $A_{1}(x)=\frac{1}{\beta[1-G(x)]} \sum_{j=0}^{\infty} \sum_{i=0}^{j} A_{k: i}\left[\frac{\Gamma_{(j+1) \theta x^{\beta}}^{c}\left(i+\frac{r}{\beta}+1\right)}{[(j+1) \theta]^{\left(i+\frac{r}{\beta}+1\right)}}+\frac{\Gamma_{(j+1) \theta x^{\beta}}^{c}{ }^{\left(i+\frac{r}{\beta}+2\right)}}{[(j+1) \theta]^{\left(i+\frac{r}{\beta}+2\right)}}\right]$

$B(x)=g(x)$ and $A_{k: i}$ is given before

if and only if $g(x)=\sum_{k, j=0}^{\infty} \sum_{i=0}^{j} A_{k: i}\left(1+x^{\beta}\right) x^{\beta(i+1)-1} e^{-(j+1) \theta x^{\beta}}$

\section{Proof.}

We have

$$
\begin{aligned}
g(x) h_{1}(x) & =\int_{x}^{\infty} u^{r} g(u) d u \\
& =\frac{1}{\beta[1-G(x)]} \sum_{k, j=0}^{\infty} \sum_{i=0}^{j} A_{k: i}\left[\frac{\Gamma_{(j+1) \theta x^{\beta}}^{c}\left(i+\frac{r}{\beta}+1\right)}{[(j+1) \theta]^{\left(i+\frac{r}{\beta}+1\right)}}+\frac{\Gamma_{(j+1) \theta x^{\beta}}^{c}\left(i+\frac{r}{\beta}+2\right)}{[(j+1) \theta]^{\left(i+\frac{r}{\beta}+2\right)}}\right] \\
& =A_{1}(x)
\end{aligned}
$$

where $\Gamma_{\alpha}(\beta)=\int_{\alpha}^{\infty} x^{\beta-1} e^{-x} d x$

Thus $h_{1}(x)=\frac{A_{1}(x)}{g(x)}$

Suppose that $h_{1}(x)=\frac{A_{1}(x)}{g(x)}$, then 


$$
\begin{aligned}
h_{1}^{\prime}(x) & =-x^{r}+\frac{A_{1}(x) g^{\prime}(x)}{(g(x))^{2}} \\
& =-x^{r}+h_{1}(x) \frac{\sum_{k, j=0}^{\infty} \sum_{i=0}^{j} A_{k: i}\left(x^{\beta(i+1)-1} x^{\beta(i+1)-2}+\left(x^{\beta(i+2)-1}\right) x^{\beta(i+2)-2}-(j+1) \theta \beta x^{\beta-1}\right) e^{-(j+1) \theta x^{\beta}}}{\sum_{k, j=0}^{\infty} \sum_{i=0}^{j} A_{k: i}\left(x^{\beta(i+1)-1}+x^{\beta(i+2)-1}\right) e^{-(j+1) \theta x^{\beta}}}
\end{aligned}
$$

Thus

$$
\frac{x^{r}+h_{1}(x)}{h_{1}(x)}=\frac{\sum_{k, j=0}^{\infty} \sum_{i=0}^{j} A_{k: i}\left(x^{\beta(i+1)-1} x^{\beta(i+1)-2}+\left(x^{\beta(i+2)-1}\right) x^{\beta(i+2)-2}-(j+1) \theta \beta x^{\beta-1}\right) e^{-(j+1) \theta x^{\beta}}}{\sum_{k, j=0}^{\infty} \sum_{i=0}^{j} A_{k: i}\left(x^{\beta(i+1)-1}+x^{\beta(i+2)-1}\right) e^{-(j+1) \theta x^{\beta}}}
$$

Integrating both sides of the above equation we obtain

$$
\begin{aligned}
g(x)= & c \exp \int\left(\frac{\sum_{k, j=0}^{\infty} \sum_{i=0}^{j} A_{k: i}\left(x^{\beta(i+1)-1} x^{\beta(i+1)-2}+\left(x^{\beta(i+2)-1}\right) x^{\beta(i+2)-2}-(j+1) \theta \beta x^{\beta-1}\right) e^{-(j+1) \theta x^{\beta}}}{\sum_{k, j=0}^{\infty} \sum_{i=0}^{j} A_{k: i}\left(x^{\beta(i+1)-1}+x^{\beta(i+2)-1}\right) e^{-(j+1) \theta x^{\beta}}}\right) d x \\
& =c \sum_{k, j=0}^{\infty} \sum_{i=0}^{j} A_{k: i}\left(x^{\beta(i+1)-1}+x^{\beta(i+2)-1}\right) e^{-(j+1) \theta x^{\beta}}, \text { where } \mathrm{c} \text { is a constant }
\end{aligned}
$$

and

$$
\frac{1}{c}=\int_{0}^{\infty} \sum_{k, j=0}^{\infty} \sum_{i=0}^{j} A_{k: i}\left(x^{\beta(i+1)-1}+x^{\beta(i+2)-1}\right) e^{-(j+1) \theta x^{\beta}}
$$

\section{Estimation of the Parameters}

In this section we introduce the method of likelihood to estimate the parameters. The maximum likelihood estimators (MLEs) for the parameters of the geometric power Lindley Poisson distribution $\operatorname{GPLP}(\lambda, \theta, \delta, \beta)$ is discussed in this section. Consider the random sample $\mathrm{x}_{1}, \mathrm{x}_{2}, \ldots, \mathrm{x}_{\mathrm{n}}$ of size $\mathrm{n}$ from GPLP $(\lambda, \theta, \delta, \beta)$ with probability density function in (6), then the likelihood function can be expressed as follows

$$
\ell=\frac{\lambda^{n} \theta^{2 n}(1-\delta)^{n}\left(1-e^{-\lambda}\right)^{n} \beta^{n} \prod_{i=1}^{n} x_{i}^{\beta-1} \prod_{i=1}^{n}\left(1+x_{i}^{\beta}\right) \exp \left(-\theta \sum_{i=1}^{n} x_{i}^{\beta}-\lambda \sum_{i=1}^{n}\left(\frac{\theta+1+\theta x_{i}^{\beta}}{1+\theta}\right) e^{-\theta x_{i}}\right)}{(1+\theta)^{n} \prod_{i=1}^{n}\left\{1-e^{-\lambda}-\delta\left[1-\exp \left(-\lambda\left(\frac{\theta+1+\theta x_{i}^{\beta}}{1+\theta}\right) e^{-\theta x_{i}^{\beta}}\right)\right]\right\}^{2}}
$$

Hence, the log-likelihood function, $\mathcal{L}$, becomes: 
M. M. Mansour et al.

$$
\begin{aligned}
\mathcal{L}=n \ln \lambda+2 n \ln \theta+n \ln (1-\delta)+n \ln \left(1-e^{-\lambda}\right)+n \ln \beta+\sum_{i=1}^{n}(\beta-1) \ln x_{i} \\
-n \ln (1+\theta)+\sum_{i=1}^{n}\left(1+x_{i}^{\beta}\right)-\theta \sum_{i=1}^{n} x_{i}^{\beta}-\lambda \sum_{i=1}^{n}\left(\frac{\theta+1+\theta x_{i}^{\beta}}{1+\theta}\right) e^{-\theta x_{i}^{\beta}} \\
-2 \sum_{i=1}^{n} \ln \left[1-e^{-\lambda}-\delta\left[1-\exp \left(-\lambda\left(\frac{\theta+1+\theta x_{i}^{\beta}}{1+\theta}\right) e^{-\theta x_{i}^{\beta}}\right)\right]\right]
\end{aligned}
$$

Therefore, the MLEs of $\lambda, \theta, \delta$ and $\beta$ must satisfy the following equations:

$$
\begin{aligned}
& \frac{\partial \mathcal{L}}{\partial \lambda}=\frac{n}{\lambda}+\frac{n}{e^{\lambda}-1}-\sum_{i=1}^{n}\left(\frac{\theta+1+\theta x_{i}^{\beta}}{1+\theta}\right) e^{-\theta x_{i}^{\beta}} \\
& -2 \sum_{i=1}^{n} \frac{e^{-\lambda}-\delta\left(\frac{\theta+1+\theta x_{i}^{\beta}}{1+\theta}\right) \exp \left(-\theta x_{i}^{\beta}-\lambda\left(\frac{\theta+1+\theta x_{i}^{\beta}}{1+\theta}\right) e^{-\theta x_{i}^{\beta}}\right)}{\left[1-e^{-\lambda}-\delta\left[1-\exp \left(-\lambda\left(\frac{\theta+1+\theta x_{i}^{\beta}}{1+\theta}\right) e^{-\theta x_{i}^{\beta}}\right)\right]\right]} \\
& \frac{\partial \mathcal{L}}{\partial \theta}=\frac{2 n}{\theta}-\frac{n}{1+\theta}-\sum_{i=1}^{n} x_{i}^{\beta}+\frac{\lambda \theta}{(1+\theta)^{2}} \sum_{i=1}^{n}\left[(2+\theta) x_{i}^{\beta}+(1+\theta) x_{i}^{\beta^{2}}\right] e^{-\theta x_{i}^{\beta}} \\
& -\sum_{i=1}^{n} \frac{2 \lambda \theta \delta \exp \left(-\theta x_{i}^{\beta}-\lambda\left(\frac{\theta+1+\theta x_{i}^{\beta}}{1+\theta}\right) e^{-\theta x_{i}^{\beta}}\right)\left[(2+\theta) x_{i}^{\beta}+(1+\theta) x_{i}^{\beta^{2}}\right]}{(1+\theta)^{2}\left[1-e^{-\lambda}-\delta\left[1-\exp \left(-\lambda\left(\frac{\theta+1+\theta x_{i}^{\beta}}{1+\theta}\right) e^{-\theta x_{i}^{\beta}}\right)\right]\right]}, \\
& \frac{\partial \mathcal{L}}{\partial \pi}=\frac{-n}{(1-\delta)}+2 \sum_{i=1}^{n} \frac{1-\exp \left(-\lambda\left(\frac{\theta+1+\theta x_{i}^{\beta}}{1+\theta}\right) e^{-\theta x_{i}^{\beta}}\right)}{1-e^{-\lambda}-\delta\left[1-\exp \left(-\lambda\left(\frac{\theta+1+\theta x_{i}^{\beta}}{1+\theta}\right) e^{-\theta x_{i}^{\beta}}\right)\right]}
\end{aligned}
$$

and

$$
\begin{aligned}
\frac{\partial \mathcal{L}}{\partial \beta}= & \frac{n}{\beta}+\sum_{i=1}^{n} \ln x_{i}+(1-\theta) \sum_{i=1}^{n} x_{i}^{\beta} \ln x_{i}-\left(\frac{\theta x_{i}^{\beta}-\theta}{1+\theta}\right) \exp \left(-\theta x_{i}^{\beta} \theta x_{i}^{\beta} \ln x_{i}\right) \\
& -2 \sum_{i=1}^{n} \frac{\pi \exp \left(-\lambda\left(\frac{\theta+1+\theta x_{i}^{\beta}}{1+\theta}\right) e^{-\theta x_{i}^{\beta}}\right)\left(\frac{\theta x_{i}^{\beta}-\theta}{1+\theta}\right) \exp \left(-\theta x_{i}^{\beta} \theta x_{i}^{\beta} \ln x_{i}\right)}{\left[1-e^{-\lambda}-\delta\left[1-\exp \left(-\lambda\left(\frac{\theta+1+\theta x_{i}^{\beta}}{1+\theta}\right) e^{-\theta x_{i}^{\beta}}\right)\right]\right]}
\end{aligned}
$$


The maximum likelihood estimator $\underline{\hat{v}}=(\hat{\lambda}, \hat{\theta}, \hat{\delta}, \hat{\beta})$ of $\underline{\vartheta}=(\lambda, \theta, \delta, \beta)$ is obtained by solving the nonlinear system of equations (17) through (20). It is usually more convenient to use nonlinear optimization algorithms such as quasi-Newton algorithm to numerically maximize the log-likelihood function.

\section{References}

[1] M. Ahsanullh, M.E. Ghitany, and D.K. Al-Mutairi, Characterizations of Lindley distribution by truncated moments. Accepted for publication in Communications in Statistics-Theory and Methods (2016).

[2] H. S. Bakouch, B. M. Al-Zahrani, A. A. Al-Shomrani, V. A. A. Marchi and F. Louzada, An extended Lindley distribution. J. Korean Stat. Soc. 41(1) (2012), 75-85.

[3] T. Bjerkedal, Acquisition of Resistance in Guinea Pies infected with Different Doses of Virulent Tubercle. (1960)

[4] M.E. Ghitany, D. K. Al-Mutairi and S. Nadarajah, Zero-truncated Poisson-Lindley distribution and its Applications. Math. Comput. Simul. 79(3) (2008), 279-287.

[5] M. E. Ghitany, D. K. Al-Mutairi, N. Balakrishnan, and L. J. Al-Enezi, Power Lindley distribution and associated inference. Computational Statistics \& Data Analysis, 64 (2013), 20-33.

[6] W. Gui, S. Zhangb and X. Lu, The Lindley-Poisson distribution in lifetime analysis and its properties. HACETTEPE JOURNAL OF MATHEMATICS AND STATISTICS, 43(6) (2014), 1063-1077.

[7] E. Mahmoudi, and H. Zakerzadeh, Generalized poisson-lindley distribution. Communications in Statistics-Theory and Methods, 39(10) (2010), 1785-1798.

[8] M. M. Mansour and S. M. Mohamed, A New Generalized of Transmuted Lindley Distribution. Applied Mathematical Sciences, 9(55) (2015), 2729-2748.

[9] F. Merovci, Transmuted lindley distribution. International Journal of Open Problems in Computer Science \& Mathematics, 6 (2013).

[10] S. Nadarajah, H. S. Bakouch, and R. Tahmasbi, A generalized Lindley distribution. Sankhya B, 73(2) (2011), 331-359.

[11] S. Nadarajah, V. G. Cancho, and E. M. Ortega, The geometric exponential Poisson distribution. Statistical Methods \& Applications, 22(3) (2013), 355-380.

[12] A. Renyi, On measures of entropy and information, Proceedings of the 4thBerkeley Symposium on Mathematical Statistics and Probability, Berkeley(CA). (University of California Press, I, 547 - 561, 1960)

[13] M. Sankaran, The discrete Poisson-Lindley distribution. Biometrics, (1970) pp. 145-149.

[14] R. Shanker, S. Sharma and R. Shanker, A two-parameter Lindley distribution for modeling waiting and survival times data. Applied Mathematics, 4(02) (2013), 363.

[15] E.A. Shannon, A Mathematical Theory of Communication, The Bell System Technical Journal, 27(10) (1948), 379 - 423.

[16] H. Zakerzadah and A. Dolati, Generalized Lindley distribution. J. Math. Ext. 3(2) (2010),13-25.

[17] H. Zakerzadeh and E. Mahmoudi, A new two parameter lifetime distribution: model and properties. (arXiv preprint arXiv:1204.4248., 2012) 